\title{
Dynamics of vortex shells in mesoscopic superconducting Corbino disks
}

\author{
V. R. Misko and F. M. Peeters \\ Department of Physics, University of Antwerpen, Groenenborgerlaan 171, B-2020 Antwerpen, Belgium
}

(Received 17 October 2006; published 14 November 2006)

\begin{abstract}
In mesoscopic superconducting disks vortices form shell structures as recently observed in $\mathrm{Nb}$ disks. We study the dynamics of such vortices, driven by an external current $I_{0}$, in a Corbino setup. At very low $I_{0}$, the system exhibits rigid-body rotation while at some critical current $I_{c, i}$ vortex shells rotate separately with angular velocities $\omega_{i}$. This critical current $I_{c, i}$ has a remarkable nonmonotonous dependence on the applied magnetic field which is due to a dynamically induced structural transition with a rearrangement of vortices over the shells similar to the Coster-Kronig transition in hollow atoms. Thermally activated externally driven flux motion in a disk with pinning centers explains experimentally observed $\omega_{i}$ as a function of $I_{0}$ and $T$ and the dynamically induced melting transition.
\end{abstract}

DOI: $10.1103 /$ PhysRevB.74.174507

\section{INTRODUCTION}

A mesoscopic Corbino disk is a unique system to study the dynamics of self-organized vortex matter in small-size superconductors. The interplay between the vortex-vortex and vortex-boundary interactions in mesoscopic superconductors leads to shape-induced giant vortex states, ${ }^{1}$ concentric shells of vortices and symmetry-induced vortexantivortex "molecules" in mesoscopic squares and triangles. ${ }^{2}$ In the Corbino geometry ${ }^{3}$ an applied current creates a gradient in the current density and thus the Lorentz force, i.e., introducing a shear driving force between the rings of vortices. This gives us the unique opportunity to study various dynamical effects related to vortex motion, e.g., the transition from elastic to plastic motion, channeling, ${ }^{4}$ vortex friction, ${ }^{5}$ etc. The dynamics of self-organized vortex matter in mesoscopic disks has many common features to, e.g., atomic matter, charged particles in Coulomb crystals, vortices in rotating Bose-Einstein condensates, magnetic colloids, synthetic nanocrystals, etc., ${ }^{6-8}$ or even large charged balls diffusing in macroscopic Wigner rings ${ }^{9}$ and can provide us with a deeper understanding of, e.g., the microscopic nature of friction, transport, magnetic, optical and mechanical properties of various physical and biological systems.

In a Corbino disk, the applied current is injected at the center and removed at the perimeter (see Fig. 1) to induce a radial current density $J$ that decays as $1 / \rho$ along the radius. ${ }^{3,10}$ As a result, vortices near the center of the disk experience a stronger Lorentz force $F_{L}$ than those near the disk's edge. For small $J$, the local shear stress is small and the whole vortex pattern moves as a rigid body. Larger $J$ result in a strong spatially inhomogeneous stress that breaks up the vortex solid and concentric annular regions move with different angular velocities. The voltage profiles measured in experiments $^{3}$ reflect different dynamical phases (elastic motion, shear-induced plastic slip) of vortex motion. The onset of plasticity in large Corbino disks was theoretically analyzed within a continuous model in Ref. 11. Within molecular dynamics (MD) simulations of interacting vortices at $T=0$, the nucleation and motion of dislocations in the vortex lattice was studied in Ref. 12.

Recently, using the Bitter decoration technique, the direct observation of rings of vortices for mesoscopic superconducting $\mathrm{Nb}$ disks was reported. ${ }^{13}$ For vorticities $L=0$ to 40 ,
PACS number(s): 74.78.Na, 74.25.Qt, 74.25.Sv

the circular symmetry led to the formation of concentric shells of vortices, similar to electron shells in atoms or in nanoclusters. ${ }^{14}$ The analysis of shell filling revealed "magicnumber" configurations (MNC) (Refs. 15-17) corresponding to a commensurability between the shells which occurs when the numbers of vortices of each shell have a common divider. ${ }^{15}$

Here, we study the dynamics of vortex shells in mesoscopic Corbino disks. Our system has the added flexibility that we have several experimentally accessible tuning parameters as, e.g., the driving shear force, the separation between the vortex rings (through the external magnetic field) and the commensurability between the vortex rings (through the relative number of vortices in each ring). In mesoscopic Corbino disks, we reveal a nonmonotonous dependence of the critical current $I_{c}$ separating two dynamical regimes, a "rigid body" or separate shell rotation, on the magnetic field $h$, and the appearance of dynamical instabilities associated with a jump in $I_{c}(h)$. We show that this unusual behavior is related to a "structural transition," i.e., an

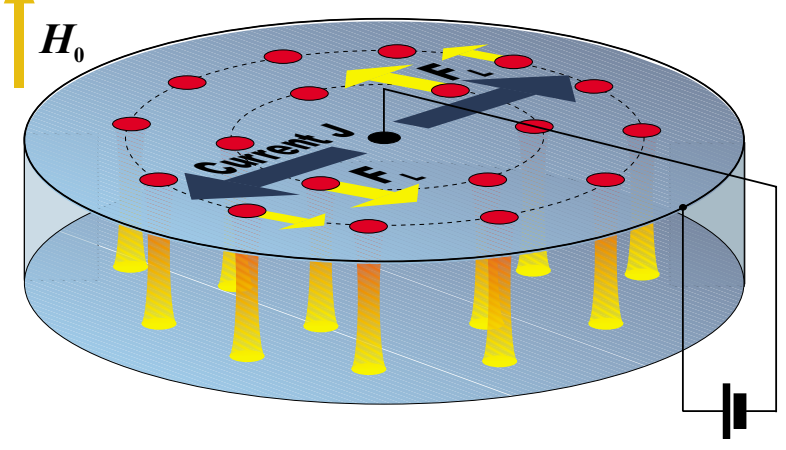

FIG. 1. (Color online) The Corbino setup: the applied current is injected at the center and removed at the perimeter of the disk to induce a radial current density $J$ (shown by dark blue/dark grey arrows) that decays as $1 / \rho$ along the radius. As a result, vortices near the center of the disk experience a stronger Lorentz force $F_{L}$ (shown by yellow/light grey arrows) than those near the disk's edge. The vortices are shown by red-to-yellow/grey-to-light grey tubes and by red/grey spots on the surface. The direction of the external applied magnetic field $H_{0}$, which is perpendicular to the surface of the disk, is shown by dark yellow/grey arrow. 
intershell vortex transition. For nonzero temperature, thermally activated externally driven flux motion is investigated, and we explain the observed two-step melting transition in Corbino disks. ${ }^{3}$

\section{THEORY AND SIMULATION}

We place a Corbino disk which has thickness $d$ and radius $R$ in a perpendicular external magnetic field $\boldsymbol{H}_{0}$. The Corbino setup is shown in Fig. 1. An external current flows radially from the center to the edge of the disk and results in the inhomogeneous sheath current density $J(\rho)=I_{\text {ext }} / 2 \pi \rho$, which makes vortices closer to the center feel a stronger force compared to the ones near the edge. The Lorentz force (per unit length) acting on vortex $i, \Phi_{0} j \times \hat{z}$, resulting from the external current is

$$
\boldsymbol{f}_{i}^{J}=\frac{\Phi_{0} I_{\mathrm{ext}}}{2 \pi} \frac{\boldsymbol{\rho}_{i} \times \hat{z}}{\rho_{i}^{2}}=f_{0} I_{0} \frac{\boldsymbol{r}_{i} \times \hat{z}}{r_{i}^{2}},
$$

where $\boldsymbol{\rho}_{i}$ is the vortex position, $\boldsymbol{r}_{i}=\boldsymbol{\rho}_{i} / R$, and $\hat{z}$ is the unit vector along the magnetic field direction which is taken perpendicular to the disk. Here $f_{0}=\Phi_{0}^{2} / 2 \pi \mu_{0} R \lambda^{2}$ $=4 \pi \mu_{0} \xi^{2} H_{c}^{2} / R$ is the unit of force, $I_{0}=\mu_{0} \Lambda I_{\text {ext }} / \Phi_{0}$, and $\Lambda$ $=\lambda^{2} / d$.

In a thin superconducting disk such that $d<\xi \ll R \ll \Lambda$, the vortex-vortex interaction force $\boldsymbol{f}_{i}^{v v}$ and the force of the vortex interaction with the shielding currents and with the edge $f_{i}^{5}$ can be modelled, respectively, by ${ }^{18-20}$

$$
\begin{gathered}
\boldsymbol{f}_{i}^{v v}=f_{0} \sum_{i, k}^{L}\left(\frac{\boldsymbol{r}_{i}-\boldsymbol{r}_{k}}{\left|\boldsymbol{r}_{i}-\boldsymbol{r}_{k}\right|^{2}}-r_{k}^{2} \frac{r_{k}^{2} \boldsymbol{r}_{i}-\boldsymbol{r}_{k}}{\left|r_{k}^{2} \boldsymbol{r}_{i}-\boldsymbol{r}_{k}\right|^{2}}\right), \\
\boldsymbol{f}_{i}^{s}=f_{0}\left(\frac{1}{1-r_{i}^{2}}-h\right) \boldsymbol{r}_{i},
\end{gathered}
$$

where $h=\pi R^{2} \mu_{0} H_{0} / \Phi_{0}=\left(H_{0} / 2 H_{c 2}\right)(R / \xi)^{2}, \boldsymbol{r}_{i}$ is the position of the $i$ th vortex, and $L$ is the number of vortices, or the vorticity. Our numerical approach is based on the Langevin dynamics algorithm, where the time integration of the equations of motion is performed in the presence of a random thermal force. The overdamped equations of motion becomes

$$
\eta \boldsymbol{v}_{i}=\boldsymbol{f}_{i}=\boldsymbol{f}_{i}^{v v}+\boldsymbol{f}_{i}^{v p}+\boldsymbol{f}_{i}^{T}+\boldsymbol{f}_{i}^{d}+\boldsymbol{f}_{i}^{s} .
$$

Here $f_{i}^{d}=f_{i}^{J}$ is the driving force [Eq. (1)], $f_{i}^{v p}$ is the force due to vortex-pin interactions, ${ }^{21}$ and $\boldsymbol{f}_{i}^{T}$ is the thermal stochastic force, obeying the fluctuation dissipation theorem

$$
\left\langle f_{\alpha, i}(t) f_{\beta, j}\left(t^{\prime}\right)\right\rangle=2 \eta \delta_{\alpha \beta} \delta_{i j} \delta\left(t-t^{\prime}\right) k_{B} T,
$$

where greek and italic indices refer to vector components and vortex labels, $\eta$ is the viscosity. The ground state of the system is obtained by simulating field-cooled experiments. ${ }^{22}$

\section{VORTEX DYNAMICS IN TWO-SHELL CORBINO DISK}

First, we consider the smallest mesoscopic Corbino disk which shows the main physics, and it has $L=19$ vortices which form the MNC $(1,6,12)$, as shown in the left-hand
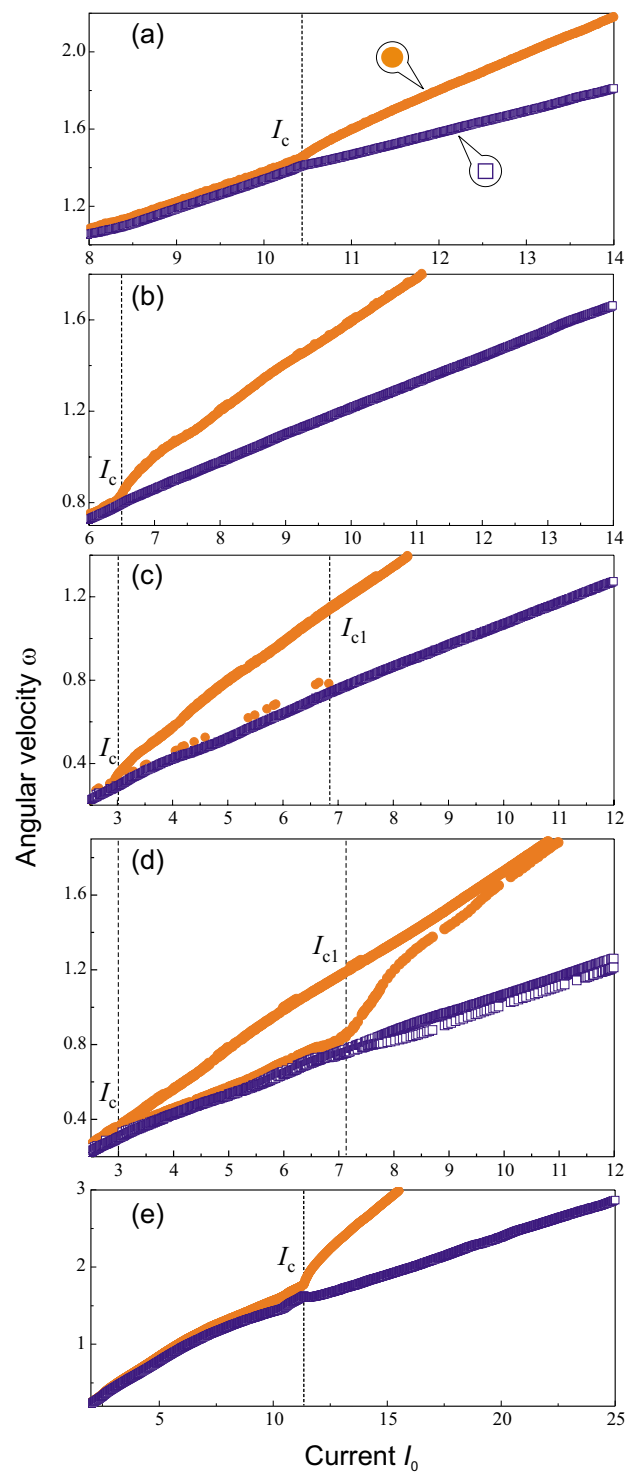

FIG. 2. (Color online) The angular velocities $\omega_{1}$ (orange/light grey solid circles) and $\omega_{2}$ (blue/dark grey open squares), measured in units of $\omega_{0}=4 \pi \mu_{0} H_{c}^{2} \xi^{2} / \eta R^{2}$, of vortices in the first and second shells, respectively, as a function of applied current $I_{0}$, measured in units of $\mu_{0} \Lambda I_{\text {ext }} / \Phi_{0}$, for $L=19$ and different magnetic fields $h$ : (a) $h=24$, a rigid-body rotation for $I_{0}<I_{c} \approx 10.4$; the shells rotate separately for $I_{0}>I_{c}$; (b) $h=25$, the critical current decreases, $I_{c} \approx 6.5$; (c) $h=26.25$, the $I_{c}$ further decreases, $I_{c} \approx 3$, and the motion becomes unstable; (d) $h=26.5$, bistable motion, a second critical current $I_{c 1} \approx 7.1$ appears; (e) $h=30$, the motion stabilizes, and first critical current $I_{c}$ disappears, and critical current $I_{c}=I_{c 1}$.

inset of Fig. 3. At weak applied magnetic field $h=24$ all the vortices are packed in an almost perfect triangular Abrikosov lattice. (Here we assume a perfect disk with no pinning, and temperature is set to zero after the "annealing" process.) We apply an increasing external current $I_{0}$, and we study the average angular velocity $\omega_{i}$ of vortices in each shell. For small $I_{0}$, the shear produced by the gradient of the Lorentz force is insufficient to break the vortex lattice. It produces only elastic deformations in the lattice which rotates as a rigid body with angular velocity 


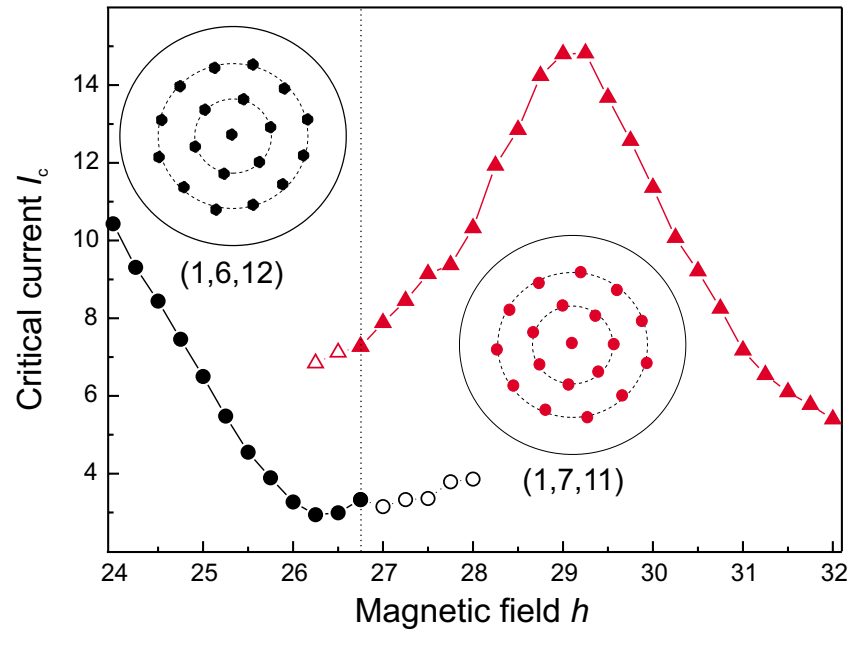

FIG. 3. (Color online) The critical current $I_{c}$ (in units of $\left.\mu_{0} \Lambda I_{\text {ext }} / \Phi_{0}\right)$ versus $h$ [in units of $\left.\left(H_{0} / 2 H_{c 2}\right)(R / \xi)^{2}\right]$ near the structural transition between the states $(1,6,12)$ (solid black circles) and $(1,7,11)$ (solid red/grey triangles), insets. Empty symbols correspond to bistable vortex motion, with two critical currents, $I_{c}$ and $I_{c 1}$. The jump in $I_{c}$ is related to an intershell vortex transition from a higher to a lower orbit, similar to the Coster-Kronig transition in hollow atoms.

$$
\omega_{\mathrm{RB}}=I / 2 \pi\left\langle r^{2}\right\rangle,
$$

where $\left\langle r^{2}\right\rangle=\sum_{i=1}^{L} r_{i}^{2} / L$ is the average square radius for the vortices in the disk.

With increasing $I_{0}$, the Lorentz force gradient reaches a critical value at which the shells start to slide with respect to each other and rotate with different velocities $\omega_{1}>\omega_{2}$. We call this the "critical current" $I_{c}$, and for the case shown in Fig. 2(a) we found $I_{c} \approx 10.4$. Now $\omega_{1}>\omega_{\mathrm{RB}}>\omega_{2}$, and the inner (outer) shell rotates faster (slower) than the rigid body. For higher applied magnetic field, e.g., $h=25$, the critical current decreases, $I_{c} \approx 6.5$ [Fig. 2(b)]. This is related to a deformation of the shells, which approach a circular shape, and therefore can more easily slide. At the same time the shells move closer to each other increasing the dynamical friction between them. At $h=26.25$, the $I_{c}$ further decreases down to $I_{c} \approx 3$, and the motion becomes unstable: for $I_{0}>3$, the inner shell rotates with angular velocity $\omega_{2}>\omega_{1}$, although at some values of $I_{0}$ in the region $3<I_{0}<6.8, \omega_{2}$ drops down to $\omega_{2}=\omega_{1}$ [Fig. 2(c)]. The appearance of this irregular motion, or "stochastization," is related to the strong dynamical friction between the shells, which can even "lock in" the shells at some values of $I_{0}$. A further increase of the magnetic field, $h=26.5$ [Fig. 2(d)], results in bistable motion of the shells: a second critical current $I_{c 1} \approx 7.1$ appears, and for currents $I_{c}<I_{0}<I_{c 1}$ the system either rotates as a rigid body or the shells rotate separately. Finally, at even higher field, e.g., $h=30$, the upper branch (corresponding to higher angular velocity) of the function $\omega\left(I_{0}\right)$ for the inner shell [shown by orange/light grey dotted line in Fig. 2(d)] disappears. Therefore, the first critical current $I_{c}$ also disappears, and the motion stabilizes with the only critical current $I_{c}=I_{c 1}[$ Fig. 2(e)]. The nonmonotonous change of the critical current $I_{c}$ with increasing applied magnetic field $h$ is summarized in Fig. 3. First, $I_{c}$ decreases, then instabilities develop in the system which in general are indicative of, and precede, a phase transition. The transition to a second critical field occurs through a bistable state characterized by two critical currents in the system. This unusual behavior can be understood by analyzing the critical current together with the structure of the vortex shells in the region of this "phase transition." The vortex patterns for magnetic field before (low fields) and after (high fields) the transition are presented on the left-hand side and on the right-hand side insets of Fig. 3. The jump in the critical current is correlated to a "structural transition" in the system where the distribution of vortices over the shells is altered, i.e., there is an intershell vortex transition from a higher orbit to a lower one, which is similar to the Coster-Kronig transition in hollow atoms (see, e.g., Ref. 23). This structural transition is accompanied by a local redistribution of the flux inside the disk, or by the appearance of flux jump instabilities, which might have an additional triggering mechanism caused by the viscosity.

We found that this jump in $I_{c}$ is also observed for other vortex configurations and is generic for vortex structures confined in Corbino disk. For instance, in a disk with $L=22$ it is related to the transition between the states $(1,8,13)$ and $(2,8,12)$ at $h=33.5$. Note that a similar behavior (a jump in the mobility) is also found in the Frenkel-Kontorova model for the locked-to-sliding transition for chains moving on commensurate potentials. ${ }^{24}$

\section{THERMALLY ACTIVATED EXTERNALLY DRIVEN DYNAMICS IN A CORBINO DISK}

Consider now a Corbino disk containing a larger number of vortex shells, e.g., $L=37$ where vortices form three shells. Note that the chosen vorticity allows the triangular-lattice MNC $(1,6,12,18)$ shown in the inset of Fig. 4. As in the above case of two shells, for small $I_{0}$ the system displays a rigid-body rotation. However, the distance between the inner and the outer shells is larger, and, thus, the Lorentz force gradient results in a stronger elastic deformation of the vortex configuration as compared to the two-shells case. The shear stress is stronger close to the center of the disk, and as a result, the inner shell splits off first at $I_{c 12}$, with increasing $I_{0}$, while second and third shells still rotate with the same angular velocity up to some critical current value $I_{c 23}>I_{c 12}$ (see Fig. 4).

The three-shell system displays a number of intershell vortex transitions when changing the applied magnetic field. Similar to the case of two shells, a structural "phase transition" occurs where vortices from a higher shell transit to a lower shell when the magnetic field increases. This leads to nonmonotonic dependences of the critical currents $I_{c 12}$ and $I_{c 23}$ on $h$ and to instabilities in the functions $\omega_{i}\left(I_{0}\right)$ at different $h$.

Now we will vary the temperature $T$ and study the thermally activated externally driven dynamics of vortices in the shells. For this purpose, we introduce pinning centers randomly distributed over the disk (for brevety we assume dense narrow pinning centers with maximum pinning force 


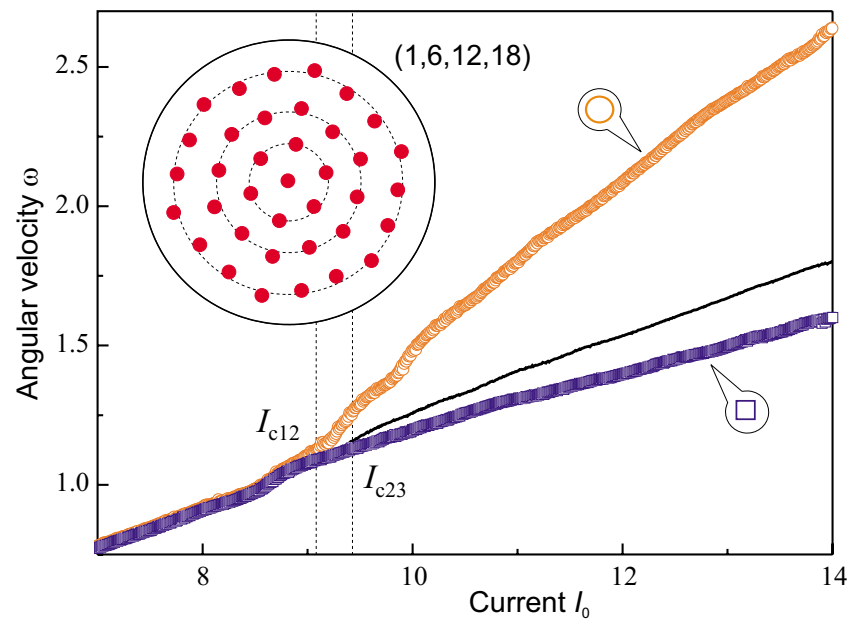

FIG. 4. (Color online) The angular velocities $\omega_{i}$ (in units of $\omega_{0}=4 \pi \mu_{0} H_{c}^{2} \xi^{2} / \eta R^{2}$ ) versus $I_{0}$ (in units of $\mu_{0} \Lambda I_{\text {ext }} / \Phi_{0}$ ) in first (orange/light grey open circles), second (black solid line), and third (blue/dark grey open squares) shells, for a three-shell vortex configuration with $L=37$. The inset shows a triangular-lattice groundstate configuration $(1,6,12,18)$ for $h=37$ at $I_{0}=0$. The critical currents are $I_{c 12} \approx 9.1$ and $I_{c 23} \approx 9.5$.

$\left.f_{p 0}\right)$ and apply a very low current $I_{0}$ that results in a Lorentz force which is less than the pinning force $f_{p 0}$ for a vortex in any shell. At low $T$ all the vortices are pinned and the shells do not move. With increasing $T$, the random-noise force is added to the (weak) Lorentz force. Intuitively one expects that vortices in the inner shell, where the Lorentz force is maximum, will depin first and start to rotate while vortices in the other shells are still pinned. However, the vortex-vortex interaction prevents this scenario, and the inner-shell vortices, if unpinned, become "trapped" by the vortex lattice itself that leads to an elastic deformation. The vortex-vortex interaction locks in the vortex configuration, and when for further increasing $T$, the onset of the motion occurs in the form of a rigid-body rotation (Fig. 5). At some temperature $T_{c 1}^{\star}$, the inner shell splits off and starts moving with a higher angular velocity, while higher shells stay locked up to a "second critical" temperature $T_{c 2}^{\star}$. The transition from a rigid body to a separate rotation of shells is called angular melting (i.e., the "vortex solid" to "vortex shells" transition). This is a multistep process which starts when the first shell splits off (i.e., at $T_{c 1}^{\star}$ in our three-shell model) and it finishes when all the shells rotate independently $\left(T \geqslant T_{c 2}^{\star}\right)$, but they keep their identity and contain a constant number of vortices with welldefined average radius $\left\langle r_{i}\right\rangle$. The radial melting is associated with a dissolving of the shells (i.e., the "vortex shells" to "vortex liquid" transition) and occurs at a higher temperature, $T_{M, r}$ (Fig. 5).

This three-shell model explains qualitatively the experimentally observed thermally induced "solid-liquid" melting transition in a Corbino disk ${ }^{3}$ with three probes (see inset to Fig. 4). Also, the behavior of $\omega_{i}\left(I_{0}\right)$ (Fig. 4) is in agreement with the experimental results. In the experiment, the superconducting disk contained a large number of vortices, and the measurements were done for rings rather than for shells. To model larger systems, we used a disk with, e.g., 123 vor-

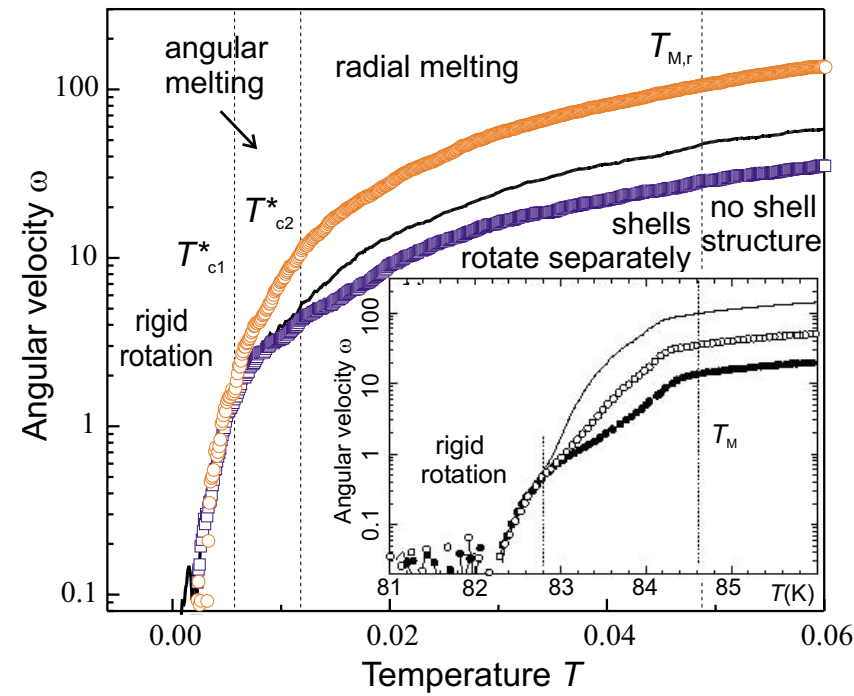

FIG. 5. (Color online) The angular velocities of vortices $\omega_{i}$ (in units of $\omega_{0}=4 \pi \mu_{0} H_{c}^{2} \xi^{2} / \eta R^{2}$ ) versus $T$ (in units of $T_{0}$ $=4 \pi \mu_{0} H_{c}^{2} \xi^{2} d / k_{B}$ ) in first, second, and third shells (using the same symbols as in Fig. 4), for $L=37, I_{0}=0.4, f_{p} / f_{0}=2.5$. The onset of motion occurs in the form of rigid-body rotation. At $T=T_{c 1}^{\star}$, the inner shell splits off, the second shell unlocks at $T=T_{c 21}^{\star}$, and shells rotate independently (angular melting). The radial melting, i.e., dissolving of the shells, occurs at $T=T_{M, r}$. Inset, the experimental $T$ dependence of $\omega_{i}$ obtained from the resistivity measurements at three different distances from the center of a Corbino disk (Ref. 3).

tices forming six shells, and we calculated average velocities in three rings (each containing two shells). We found that the results for larger disks are similar to the above three-shell system, but the radial melting temperature $T_{M, r}$ is lower, in agreement with the experiment.

\section{CONCLUSIONS}

We predict an unusual nonmonotonous behavior of the critical current for unlocking of the vortex rings with magnetic field which originates from a "structural transition" where a vortex jumps from a higher shell to a lower shell similarly to the Coster-Kronig transition in hollow atoms. Pushing the experiments of Ref. 3 into the mesoscopic regime will allow one to detect experimentally the predicted behavior. The vortex motion in the presence of pinning centers reveals the onset of a rigid-body rotation, due to thermally activated externally driven flux motion. With increasing $T$, the inner shell splits off first and subsequently all the shells start moving separately. The present numerical study explains the experiments of Ref. 3, in which vortex velocities in adjacent layers in a Corbino disk were studied as a function of $I$ and $T$.

\section{ACKNOWLEDGMENTS}

The authors thank B. Janko, F. Nori, and U. Welp for useful discussions and hospitality. This work was supported by the Flemish Science Foundation, and the Belgian Science Policy. One of the authors (V.R.M.) acknowledges partial support through POD. 
${ }^{1}$ V. A. Schweigert, F. M. Peeters, and P. S. Deo, Phys. Rev. Lett. 81, 2783 (1998); A. Kanda, B. J. Baelus, F. M. Peeters, K. Kadowaki, and Y. Ootuka, ibid. 93, 257002 (2004).

${ }^{2}$ L. F. Chibotaru, A. Ceulemans, V. Bruyndoncx, and V. V. Moshchalkov, Nature (London) 408, 833 (2000); Phys. Rev. Lett. 86, 1323 (2001); V. R. Misko, V. M. Fomin, J. T. Devreese, and V. V. Moshchalkov, ibid. 90, 147003 (2003).

${ }^{3}$ G. W. Crabtree, D. Lopez, W. K. Kwok, H. Safar, and L. M. Paulius, J. Low Temp. Phys. 117, 1313 (1999); D. Lopez, W. K. Kwok, H. Safar, R. J. Olsson, A. M. Petrean, L. Paulius, and G. W. Crabtree, Phys. Rev. Lett. 82, 1277 (1999).

${ }^{4}$ R. Besseling, P. H. Kes, T. Dröse, and V. M. Vinokur, New J. Phys. 7, 71 (2005).

${ }^{5}$ A. Maeda, Y. Inoue, H. Kitano, S. Savel'ev, S. Okayasu, I. Tsukada, and F. Nori, Phys. Rev. Lett. 94, 077001 (2005).

${ }^{6}$ T. B. Mitchell, J. J. Bollinger, W. M. Itano, and D. H. E. Dubin, Phys. Rev. Lett. 87, 183001 (2001).

${ }^{7}$ A. Pertsinidis and X. Z. Ling, Nature (London) 413, 47 (2001).

${ }^{8}$ C. Murray, C. Kagan, and M. Bawendi, Annu. Rev. Mater. Sci. 30, 545 (2000).

${ }^{9}$ G. Coupier, M. Saint Jean, and C. Guthmann, Phys. Rev. E 73, 031112 (2006).

${ }^{10}$ Y. Paltiel, E. Zeldov, Y. Myasoedov, M. L. Rappaport, G. Jung, S. Bhattacharya, M. J. Higgins, Z. L. Xiao, E. Y. Andrei, P. L. Gammel, and D. J. Bishop, Phys. Rev. Lett. 85, 3712 (2000).
${ }^{11}$ P. Benetatos and M. C. Marchetti, Phys. Rev. B 65, 134517 (2002).

${ }^{12}$ M.-C. Miguel and S. Zapperi, Nat. Mater. 2, 477 (2003).

${ }^{13}$ I. V. Grigorieva, W. Escoffier, J. Richardson, L. Y. Vinnikov, S. Dubonos, and V. Oboznov, Phys. Rev. Lett. 96, 077005 (2006).

${ }^{14}$ Y. N. Ovchinnikov and V. Z. Kresin, Eur. Phys. J. B 45, 5 (2005); 47, 333 (2005).

${ }^{15}$ V. A. Schweigert and F. M. Peeters, Phys. Rev. B 51, 7700 (1995).

${ }^{16}$ V. M. Bedanov and F. M. Peeters, Phys. Rev. B 49, 2667 (1994).

${ }^{17}$ L. J. Campbell and R. M. Ziff, Phys. Rev. B 20, 1886 (1979).

${ }^{18}$ A. I. Buzdin and J. P. Brison, Phys. Lett. A 196, 267 (1994).

${ }^{19}$ L. R. E. Cabral, B. J. Baelus, and F. M. Peeters, Phys. Rev. B 70, 144523 (2004).

${ }^{20}$ B. J. Baelus, L. R. E. Cabral, and F. M. Peeters, Phys. Rev. B 69, 064506 (2004).

${ }^{21}$ C. Reichhardt, C. J. Olson, and F. Nori, Phys. Rev. Lett. 78, 2648 (1997); Phys. Rev. B 57, 7937 (1998); Phys. Rev. B 58, 6534 (1998).

${ }^{22}$ K. Harada, O. Kamimura, H. Kasai, T. Matsuda, A. Tonomura, and V. V. Moshchalkov, Science 274, 1167 (1996).

${ }^{23}$ J. Limburg, J. Das, S. Schippers, R. Hoekstra, and R. Morgenstern, Phys. Rev. Lett. 73, 786 (1994).

${ }^{24}$ O. M. Braun, and Yu. M. Kivshar, The Frenkel-Kontorova Model: Concepts, Methods and Applications (Springer, Berlin, 2004). 